Simultaneous bilateral PCNL and stent removal in parathyroid adenoma

\title{
Images - Simultaneous bilateral percutaneous nephrolithotomy and encrusted ureteric stent removal in a patient with an ectopic parathyroid adenoma
}

Cale E. Leeson ${ }^{1}$; Fabiola Oquendo ${ }^{2}$; Hassan A. Hassan ${ }^{3}$; Scott Bonneville ${ }^{4}$; Amer Alaref ${ }^{5}$; Walid Shahrour'; Hazem M. Elmansy ${ }^{2}$

${ }^{1}$ Northern Ontario School of Medicine, Thunder Bay, ON, Canada; ${ }^{2}$ Urology, Northern Ontario School of Medicine, Thunder Bay, ON, Canada; ${ }^{3}$ Otolaryngology, Northern Ontario School of Medicine, Thunder Bay, ON, Canada; ${ }^{4}$ Anesthesia, Northern Ontario School of Medicine, Thunder Bay, ON, Canada; ${ }^{5}$ Radiology, Northern Ontario School of Medicine, Thunder Bay, ON, Canada

Cite as: Leeson CE, Oquendo F, Hassan HA, et al. Images - Simultaneous bilateral percutaneous nephrolithotomy and encrusted ureteric stent removal in a patient with an ectopic parathyroid adenoma. Can Urol Assoc J 2020 August 7; Epub ahead of print.

http://dx.doi.org/10.5489/cuaj.6568

Published online August 7, 2020

$* * *$

\section{Introduction}

Urolithiasis is a common presenting urologic complaint, with an overall prevalence in North America of $7-13 \% .{ }^{1}$ For patients with bilateral complicated stones such as staghorn calculi, simultaneous bilateral percutaneous nephrolithotomy (SB-PCNL) has emerged as an effective endourologic procedure. ${ }^{2}$ Additionally, the initial management for complications of stone disease, such as obstructing stones, may require the placement of ureteral stents, which can become encrusted and difficult to remove. ${ }^{3}$ Preliminary metabolic workup to rule out systemic disorders such as hyperparathyroidism is recommended. ${ }^{4}$ First-line treatment for hyperparathyroidism with objective renal involvement is parathyroidectomy. ${ }^{5}$ Recurrent stones after parathyroidectomy should prompt urologists to evaluate potential causes of failed parathyroidectomy, and rule out an ectopic parathyroid adenoma. ${ }^{6}$

\section{Case report}

A 68-year-old male presented to the emergency department with a two-week history of left flank pain. The patient had a previous history of right percutaneous nephrolithotomy (PCNL) and left ureteroscopy (URS), with stone analysis showing calcium phosphate. He was considered stonefree on four-week post-operative follow-up computerized tomography (CT) scan. Moreover, he had a previous history of parathyroidectomy for hyperparathyroidism, with normal parathyroid hormone (PTH) and calcium levels post-procedure.

The patient's vital signs at presentation were: heart rate, 95 beats/minute; blood pressure, 128/76 mm Hg; respiratory rate, 19 breaths/minute; and temperature, $36.9^{\circ} \mathrm{C}$. Oxygen saturation 
was $98 \%$ on room air. On physical examination, the patient appeared in distress, which improved after parenteral analgesia. Initial laboratory studies were unremarkable. Based on the patient's history and symptoms, CT scan was ordered and showed bilateral staghorn kidney stones (Figure 1). On metabolic workup, PTH and calcium levels were elevated, despite having a previous parathyroidectomy. Otolaryngology was consulted for further investigation and the patient was found to have a right-sided ectopic superior mediastinal paraesophageal parathyroid adenoma, which was thought to be the etiology of the stone recurrence. In the meantime, a left ureteric stent was inserted due to severe left renal colic. After discussion with the patient, it was decided that SB-PCNL would be performed in two weeks given the concern of stent encrustation.

During SB-PCNL, our concern was confirmed as the previously placed left ureteric stent was severely encrusted and difficult to remove. Multiple stones around the stent were fragmented with holmium laser via retrograde ureteroscopy and removed along with the stent (Figure 2,3). Bilateral ureteric catheters were then inserted, and SB-PCNL was performed. At the end of the procedure, flexible nephroscopy confirmed absence of stones bilaterally. Total operating time was 270 minutes. After the procedure, the patient was admitted to hospital overnight in stable condition. Post-operative day one CT scan showed a $5 \mathrm{~mm}$ residual stone in the right kidney. There were no residual stones in the left kidney (Figure 4).

The ureteric stents were removed via cystoscopy two weeks later, already showing significant encrustation. Stone analysis revealed calcium phosphate stones as before. One-month CT scan revealed again multiple bilateral stones, the largest measured $5 \mathrm{~mm}$ and $7 \mathrm{~mm}$ in the right and left kidney, respectively. While waiting for the ectopic parathyroidectomy, his followup CT scan at three months revealed an obstructing $16 \mathrm{~mm}$ stone in the right kidney, and a $7 \mathrm{~mm}$ non-obstructing stone in the lower pole of the left kidney. Finally, the patient underwent ectopic parathyroidectomy, after which a right PCNL was successfully performed. He recovered well, and was stone-free on CT scan in the right kidney at four weeks, three months and one-year follow-ups. The stone in the left lower pole remained unchanged as the patient decided to continue observation.

\section{Discussion}

SB-PCNL has emerged as an effective management strategy in patients with bilateral urolithiasis, given its potential to reduce total anesthesia time, overall hospital stay and the need for further procedures compared to a staged approach. ${ }^{2,7,8}$ In our case, the procedure was challenging with the added necessity of removing a severely encrusted ureteric stent. The total operative time was 270 minutes, with a post-operative CT scan showing $0 \mathrm{~mm}$ in the left kidney, and $5 \mathrm{~mm}$ in the right. To our knowledge, this is the first reported case of a SB-PCNL and encrusted stent removal with retrograde ureteroscopy and laser lithotripsy in the same operative session. The addition of an encrusted ureteric stent removal to SB-PCNL, if necessary, may be feasible for experienced surgeons in high-volume centres.

Ureteric stents can become encrusted in patients with a history of urolithiasis and with progressively longer indwelling times. ${ }^{3} \mathrm{~A}$ review of patient case series revealed most encrusted 
stents had been in place for more than three months. ${ }^{3}$ Our patient had a stent placed for two weeks before it was completely irremovable and encrusted. This may have been compounded by a previous stone history and recurrent hyperparathyroidism related to an ectopic parathyroid adenoma. Further research is required for definitive association between the degree of hyperparathyroidism and stent encrustation rates. Nevertheless, urologists should consider frequently monitoring patients with stents if there is a history of large stone burden and metabolic abnormalities such as hyperparathyroidism.

Hyperparathyroidism is a well-established risk factor for urolithiasis. In fact, the prevalence of concurrent hyperparathyroidism in patients presenting with stones is $2-8 \%{ }^{9} \mathrm{On}$ initial presentation of urolithiasis, metabolic work-up should be performed to rule out disorders such as hyperparathyroidism. ${ }^{4}$ First-line treatment is parathyroidectomy, after which the recurrence of kidney stones decreases. ${ }^{5}$ Causes of failed parathyroidectomy should be explored if elevated PTH recurs after six months, ${ }^{5}$ including an ectopic parathyroid adenoma (1-3\% of all parathyroid tumours). ${ }^{10}$ Our patient had an elevated PTH with symptomatic bilateral staghorn stones one year after parathyroidectomy, due to a rare ectopic superior paraesophageal parathyroid adenoma. The superior paraesophageal location represents $7 \%$ of cases. ${ }^{6}$ Therefore, an ectopic parathyroid adenoma should be considered as an etiology in patients with recurrent urolithiasis after parathyroidectomy.

\section{Conclusions}

SB-PCNL is an effective management strategy for extensive bilateral urolithiasis, and the addition of an encrusted ureteric stent removal in the same session may be feasible for experienced endourologists. However, to avoid stent encrustation in patients with extensive metabolic abnormalities, frequent monitoring is required. Finally, evaluation of ectopic parathyroid adenoma as a possible etiology should be considered in patients with recurrent urolithiasis after parathyroidectomy. 


\section{References}

1. Sorokin I, Mamoulakis C, Miyazawa K, et al. Epidemiology of stone disease across the world. World J Urol 2017;35:1301-20.

2. Jones P, Dhliwayo B, Rai BP, et al. Safety, feasibility, and efficacy of bilateral synchronous percutaneous nephrolithotomy for bilateral stone disease: evidence from a systematic review. J Endourol 2017;31:334-40.

3. Vanderbrink BA, Rastinehad AR, Ost MC, et al. Encrusted urinary stents: evaluation and endourologic management. J Endourol 2008;22:905-12.

4. Dion M, Ankawi G, Chew B, et al. CUA guideline on the evaluation and medical management of the kidney stone patient-2016 update. Can Urol Assoc J 2016;10:E347.

5. Wilhelm SM, Wang TS, Ruan DT, et al. The American Association of Endocrine Surgeons guidelines for definitive management of primary hyperparathyroidism. JAMA Surg 2016;151:959-68.

6. Phitayakorn R, McHenry CR. Incidence and location of ectopic abnormal parathyroid glands. Am J Surg 2006;191:418-23.

7. Williams SK, Hoenig DM. Synchronous bilateral percutaneous nephrostolithotomy. $J$ endourol 2009;23:1707-12.

8. Holman E, Munim Khan A, Pasztor I, et al. Simultaneous bilateral compared with unilateral percutaneous nephrolithotomy. BJU Int 2002;89:334-8.

9. Rejnmark L, Vestergaard P, Mosekilde L. Nephrolithiasis and renal calcifications in primary hyperparathyroidism. J Clin Endocrinol Metab 2011;96:2377-85.

10. Thompson NW, Eckhauser FE, Harness JK. The anatomy of primary hyperparathyroidism. Surgery 1982;92:814-21. 


\section{Figures and Tables}

Fig. 1. Preoperative non-contrast computed tomography (CT) scan prior to stent insertion.

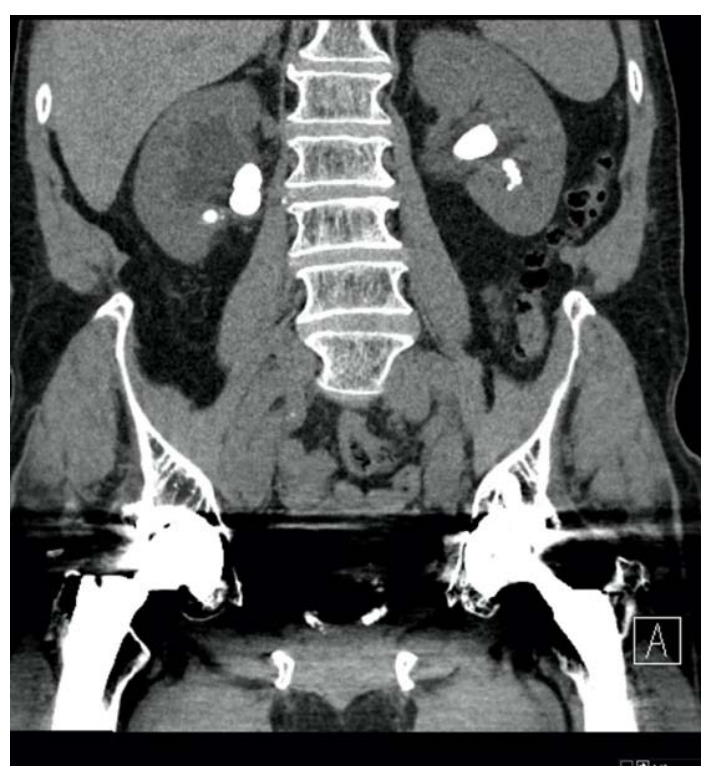

Fig. 2. Ureteroscopy with laser fragmentation of stones around the stent (arrow indicates stone attached to the stent).

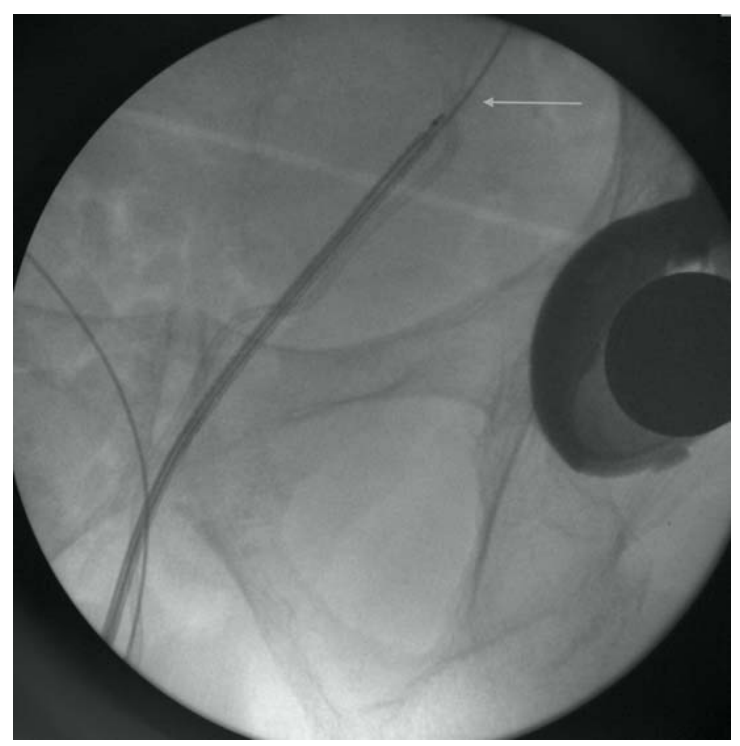


Fig. 3. Ureteroscopy with laser fragmentation of residual stones (arrow) in proximal ureter after stent removal.

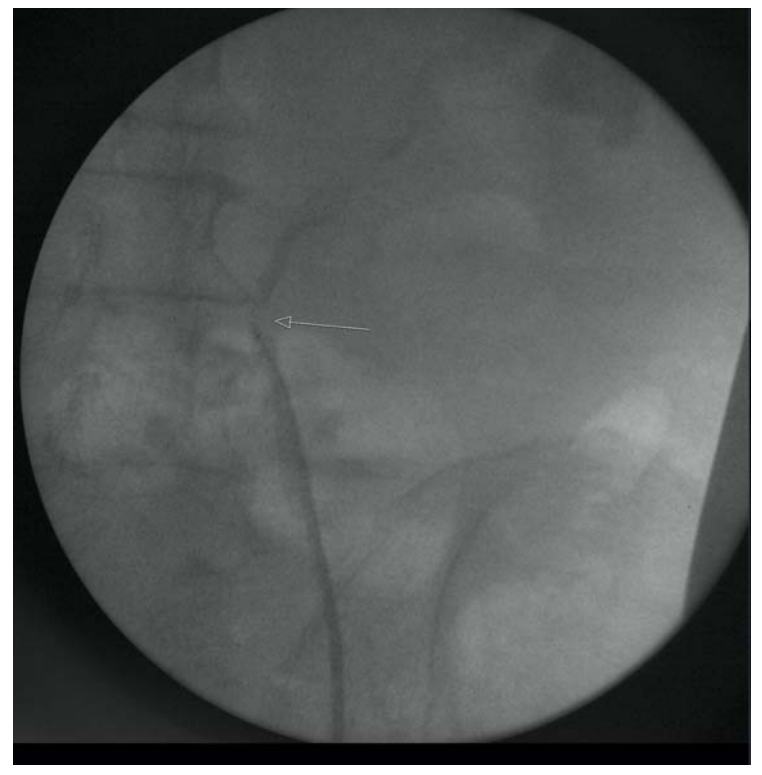

Fig. 4. Postoperative day one non-contrast computed tomography scan.

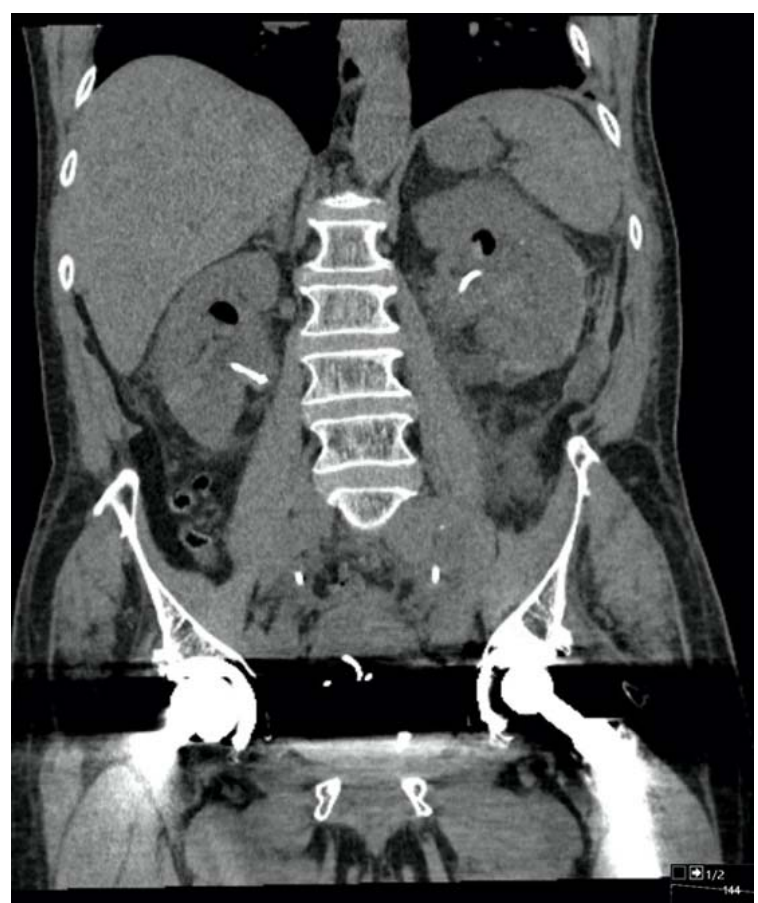

